$\begin{array}{ll}\text { Research Square } & \text { Preprints are preliminary reports that have not undergone peer review. } \\ \text { They should not be considered conclusive, used to inform clinical practice, } \\ \text { or referenced by the media as validated information. }\end{array}$

\title{
Aggresome Formation and Liquid-liquid Phase Separation Independently Induce Cytoplasmic Aggregation of TAR DNA-binding protein 43
}

Koji Yamanaka ( $\nabla$ kojiyama@riem.nagoya-u.ac.jp )

Nagoya University https://orcid.org/0000-0003-4655-0035

Seiji Watanabe

Nagoya University

Hidekazu Inami

Nagoya University

Kotaro Oiwa

Nagoya University

Yuri Murata

Nagoya University

Shohei Sakai

Nagoya University

Okiru Komine

Nagoya University

Akira Sobue

Nagoya University

Yohei Iguchi

Nagoya University

Masahisa Katsuno

Nagoya University

\section{Research article}

Keywords: Amyotrophic lateral sclerosis (ALS), Frontotemporal lobar degeneration (FTLD), TAR DNA-binding protein 43 (TDP-43), aggresome, Histone deacetylase 6 (HDAC6)

Posted Date: June 16th, 2020

DOI: https://doi.org/10.21203/rs.3.rs-28758/v1

License: (1) This work is licensed under a Creative Commons Attribution 4.0 International License. Read Full License

Version of Record: A version of this preprint was published on October 8th, 2020. See the published version at https://doi.org/10.1038/s41419-020-03116-2. 


\section{Abstract}

Background Cytoplasmic inclusion of TAR DNA-binding protein 43 (TDP-43) is a pathological hallmark of amyotrophic lateral sclerosis (ALS) and a subtype of frontotemporal lobar degeneration (FTLD). Recent studies have suggested that the formation of cytoplasmic TDP-43 aggregates is dependent on a liquidliquid phase separation (LLPS) mechanism. However, it is unclear whether TDP-43 pathology is induced through a single intracellular mechanism such as LLPS.

Methods We established a TDP-43 aggregation screening system using a cultured neuronal cell line stably expressing EGFP-fused TDP-43 and a mammalian expression library of the inherited ALS/FTLD causative genes. We performed a screening to identify the intracellular mechanisms responsible for TDP-43 aggregation. An immunofluorescence study was conducted using the sporadic ALS spinal cord sections.

Results We found that microtubule-related proteins (MRPs) and RNA-binding proteins (RBPs) co-aggregated with TDP-43. MRPs and RBPs sequestered TDP43 into the cytoplasmic aggregates through distinct mechanisms such as microtubules and LLPS, respectively. The MRPs-induced TDP-43 aggregates were co-localized with aggresomal markers and dependent on histone deacetylase 6 (HDAC6), suggesting that aggresome formation induced the co-aggregation. However, the MRPs-induced aggregates were not affected by 1,6-hexanediol, an LLPS inhibitor. On the other hand, the RBPs-induced TDP-43 aggregates were sensitive to 1,6-hexanediol, but not dependent on microtubules or HDAC6. In sporadic ALS patients, approximately half of skein-like TDP-43 inclusions were co-localized with HDAC6, but round and granular type inclusion were not. Moreover, HDAC6-positive and HDAC6-negative inclusions were found in the same ALS patient, suggesting that the two distinct pathways are both involved in TDP-43 pathology.

Conclusion Our findings suggest that at least two distinct pathways (i.e., aggresome formation and LLPS ) are involved in inducing the TDP-43 pathologies.

\section{Background}

Abnormal accumulation of TAR DNA binding protein-43 (TDP-43) is a pathological hallmark of amyotrophic lateral sclerosis (ALS), a fatal neurodegenerative disease characterized by a selective loss of motor neurons, and a subtype of frontotemporal lobar degeneration (FTLD-TDP) [1]. TDP-43 is a DNA/RNAbinding protein that is predominantly localized in nuclei and plays multifunctional roles in RNA metabolism, including pre-mRNA splicing, translational control, and mRNA stability [2, 3]. However, in most of the ALS and FTLD-TDP cases, TDP-43 is mislocalized into the cytoplasm and forms inclusion bodies [4, 5]. In addition to this, more than 50 mutations in the TARDBP gene, encoding TDP-43, have been identified as a cause of inherited ALS[6]. These observations suggest that dysfunction of TDP-43 is a significant component of ALS pathogenesis. Thus, understanding the mechanism of TDP-43 aggregation will uncover the mechanistic basis for TDP-43 pathology and neurodegeneration in ALS/FTLD.

Recently, a number of studies revealed that many kinds of RNA binding proteins (RBPs), including TDP-43, spontaneously develop granule-like structures via a liquid-liquid phase separation (LLPS) mechanism [7, 8]. LLPS is a process in which proteins and nucleotides abruptly segregate into two distinct phases, enabling the formation of intracellular membrane-less organelles [9] such as p-bodies[10] and stress granules[11, 12]. However, once excess amounts of proteins are collected via LLPS, the complexes quickly transform into pathological inclusions that are often found in neurodegenerative diseases [13, 14]. ALS causative gene products, including FUS, TIA-1, and, of course, TDP-43, are proposed to form aggregates via LLPS. Consistent with this hypothesis, recent studies have discovered that optical multimerization of cytoplasmic TDP-43 induces the aggregation and sequestration of endogenous nuclear TDP-43 into the cytoplasmic aggregates that are dependent on $\operatorname{LLPS}[15,16]$.

However, whether LLPS is solely responsible for the cytoplasmic aggregation of TDP-43 remains unclear. Among the over 25 inherited ALS/FTLD causative genes, there is a considerable number of the genes encoding the proteins that have the functions different from RNA regulation [17, 18]. For instance, SQSTM1 and TBK1 encode proteins involved in autophagy, while PFN1 and TUBA4A encode cytoskeletal proteins. Considering that TDP-43 pathology is also observed in the most of inherited ALS/FTLD patients, there may be the intracellular mechanisms other than LLPS stimulating the formation of TDP-43 aggregates in ALS/FTLD lesions.

In this study, we aimed to determine the intracellular pathways involved in the formation of TDP-43 aggregates. Using a screening system of cytoplasmic TDP43 aggregations induced by the expression of inherited ALS/FTLD causative genes, we established that, in addition to the RBPs, microtubule-related proteins (MRPs) also co-aggregated with TDP-43 in the cytoplasm. The MRPs induced co-aggregation of TDP-43 via the formation of aggresomes, cytoplasmic aggregations that are dependent on microtubules. In contrast, the RBPs-induced aggregations were dependent on LLPS. Therefore, both the TDP-43 coaggregation pathways were independent from each other. Moreover, we also showed that a subset of TDP-43 inclusions in spinal motor neurons of sporadic ALS cases were co-localized with histone deacetylase 6 (HDAC6), an aggresome marker. Our findings suggest that at least two distinct pathways (i.e., aggresome formation and LLPS) are involved in inducing the TDP-43 pathologies.

\section{Methods}

Antibodies

Antibodies used in this study are as follows; anti-HDAC6 (1:1000 for immunoblotting, 1:50 for immunohistochemistry, \#7558, RRID: AB_10891804, Cell Signaling Technology, Danvers, MA, USA), anti-y-tubulin (1:1000, \#ab27074, RRID: AB_2211240, Abcam, Cambridge, UK), anti-vimentin (1:50, \#3932, RRID: AB_2288553, Cell Signaling), anti-TDP-43 (1:1000, clone 3H8, \#MABN45, EMD Millipore, Billerica, MA), anti- $\beta$-actin (1:5000, \#5441, RRID: AB_476744, Sigma- 
Mouse neuroblastoma Neuro2a (RRID: CVCL_0470) (N2a) cells were maintained and differentiated, as described elsewhere [19]. To establish the cell line stably expressing EGFP fused human TDP-43 wild-type or nuclear localization signal (NLS) deficient mutant [20], we transfected linearized pcDNA3.1 (+) (Thermo Fisher Scientific Inc, Waltham, MA, USA) inserted each TDP-43 cDNA with Lipofectamine 2000 (Thermo Fisher). The cells were cultured for two weeks in a growth medium containing $1.0 \mathrm{~g} / \mathrm{L} \mathrm{G-418} \mathrm{(Nacalai} \mathrm{Tesque,} \mathrm{Kyoto,} \mathrm{Japan).} \mathrm{The} \mathrm{colonies} \mathrm{were} \mathrm{isolated} \mathrm{and} \mathrm{confirmed} \mathrm{the} \mathrm{expression} \mathrm{of} \mathrm{EGFP-TDP-43} \mathrm{by}$ fluorescent microscopy and immunoblotting. The established cell lines were maintained in a growth medium containing $1.0 \mathrm{~g} / \mathrm{L} \mathrm{G-418.} \mathrm{HeLa} \mathrm{(CCL-2)} \mathrm{and}$ HEK293 (CRL-1573) cells, both obtained from ATCC, were maintained in Dulbecco's Modified Eagle's' Medium (DMEM) containing $4.5 \mathrm{~g} / \mathrm{L}$ glucose supplemented with $10 \%\left(\mathrm{v} / \mathrm{v}\right.$ ) fetal bovine serum (FBS), $100 \mathrm{U} / \mathrm{mL}$ penicillin, and $100 \mu \mathrm{g} / \mathrm{mL}$ streptomycin (all from Thermo Fisher) at $37^{\circ} \mathrm{C}$ in a humidified chamber containing $5 \% \mathrm{CO}_{2}$. Total RNAs of HeLa and HEK293 cells were isolated using the RNeasy-micro kit (QIAGEN, Hilden, Germany).

Stealth siRNA against the murine Hdac6 gene was obtained from Thermo Fisher. siRNA (20 nM) was transfected into N2a cells using Lipofectamine RNAi Max (Thermo Fisher) as described by the manufacturer.

\section{TDP-43 aggregation screening using mammalian inherited ALS/FTLD causative gene expression library}

Full-length wild-type cDNA of each familial ALS causative gene was amplified using the gene-specific primers from cDNA synthesized from total RNA of HeLa or HEK293 cells. The amplified cDNA was inserted into pmCherry-N1/C1 vectors (Clontech Laboratories Inc, Mountain View, CA, USA) using SLiCE reaction [21]. ALS/FTLD-causing mutations were introduced according to the guidance of the Primestar Mutagenesis Basal Kit (Takara Bio, Shiga, Japan).

N2a cells stably expressing EGFP fused TDP-43, which is described above, were seeded at $5.0 \times 10^{4}$ / well on a 4 well cover-slide chamber (AGC Techno Glass Inc, Shizuoka, Japan) coated with poly-D-lysine. On the next day, $0.25 \mu \mathrm{g} /$ well plasmids to express inherited ALS/FTLD causative genes were transfected with Lipofectamine 2000 (Thermo Fisher) according to the manufacturer's guide. After $6 \mathrm{~h}$ of the transfection, the medium was changed into a differentiation medium (DMEM + 2\%(v/v) fetal bovine serum and 2 mM N6,2'-0-dibutyryladenosine-3',5'-cyclic monophosphate (Nacalai Tesque, Kyoto, Japan)), and cultured for $48 \mathrm{~h}$. Then, the non-fixed cells were observed using laser scanning confocal microscopy (LSM-700; Carl Zeiss AG, Oberkochen, Germany) and the equipped software (Zen; Carl Zeiss AG).

For the treatment with 1,6-hexanediol (1,6-HD) (Sigma), the medium was replaced with a fresh one with/without 4\%(v/v) 1,6-HD and incubated for 10 min at room temperature. Then, the non-fixed cells were observed using laser scanning confocal microscopy.

\section{Immunoblotting and Immunofluorescence}

Immunoblotting analyses were performed as described elsewhere [22]. For immunofluorescence analyses of $\mathrm{Y}$-tubulin and vimentin, cultured N2a cells were transfected and cultured in a differentiation medium for $48 \mathrm{~h}$. Then, the cells were fixed with ice-cold methanol at $-30^{\circ} \mathrm{C}$ for 20 min. After three times wash with TBS (50 mM Tris- $\mathrm{HCl}(\mathrm{pH} 7.4), 150 \mathrm{mM} \mathrm{NaCl})$, the cells were immunostained as described previously [22]. Briefly, the cells were incubated with primary antibodies at $4{ }^{\circ} \mathrm{C}$ for over-night, following incubation with secondary antibodies at room temperature for $2 \mathrm{~h}$. Immunofluorescence images were obtained by a confocal laser scanning microscopy (LSM-700; Carl Zeiss AG, Oberkochen, Germany) and the equipped software (Zen; Carl Zeiss AG).

\section{Postmortem Human Tissues}

Specimens of spinal cords from six patients with sporadic ALS and two control patients with other neurological diseases were obtained by autopsy with informed consent (Additional Table A1). The diagnosis of ALS was confirmed by El Escorial diagnostic criteria as defined by the World Federation of Neurology. The ethics committee approved the collection of tissues and their use in this study of Nagoya University. For immunofluorescence analyses, the sections were prepared from formalin-fixed and paraffin-embedded tissues, deparaffinized, and incubated at $90{ }^{\circ} \mathrm{C}$ for 40 min in HistVT One (Nacalai Tesque). Statistical analyses

All the data from immunofluorescence and semi-quantitative immunoblotting were analyzed by an unpaired t-test for the comparison between two groups, or one-way ANOVA followed by the posthoc Tukey's multiple comparison t-test for the comparison among more than three groups, respectively. All the statistical analyzes were carried out using GraphPad Prism software (GraphPad Software, La Jolla, CA).

\section{Results}

TDP-43 co-aggregation screening reveals the involvement of microtubule-related proteins (MRPs) and RNA-binding proteins (RBPs) in cytoplasmic TDP-43 aggregation

To identify intracellular pathways involved in cytoplasmic TDP-43 aggregation, we established a screening system for the co-aggregation of TDP-43 and ALS/FTLD-causative proteins (Fig. 1A). We generated N2a cells that stably expressed EGFP-fused wild-type or NLS-defective TDP-43 (TDP-43 ${ }^{\text {WT }}$ or TDP$43^{\triangle N L S}$, respectively) as reporter cell lines to visualize TDP-43 aggregates (Fig. 1B). When both the cells were cultured in normal conditions, no TDP-43 aggregates were observed. We also prepared an inherited ALS/FTLD-causative gene expression library (19 genes in total, see Table 1). The library consisted of expression plasmids of mCherry-fused wild-type or two independent variants carrying ALS/FTLD-linked mutations for each of the genes. Then, we transfected each plasmid into the reporter cells and observed them after two days of incubation. We then screened the library to identify the ALS/FTLD genes that induce cytoplasmic TDP-43 aggregation in N2a reporter cells that stably express EGFP-TDP-43 ${ }^{\Delta N L S}$. The screening results are summarized in Table 1. Our screening identified that $14 / 19(74 \%)$ gene products formed intracellular aggregates and 9 of them ( $9 / 19$ in total, $47 \%)$ co-aggregated with TDP-43 ${ }^{\Delta N L S}$. Representative images for the cells expressing each of the inherited ALS/FTLD causative genes are shown in Additional Figure A1. We also found that the ALS/FTLD 
causative gene products are classified into three groups: gene products that induce co-aggregates with TDP-43 (Group 1) (Fig. 1C), gene products that induce aggregates without TDP-43 (Group 2) (Fig. 1D), and gene products that do not form aggregates (Group 3) (Fig. 1E).

More importantly, all of the gene products in Group 1 induced cytoplasmic translocation and aggregation of TDP-43 WT (Fig. 2A, Additional Fig. A2). Interestingly, the Group 1 genes were involved in either microtubular dynamics or RNA regulation, suggesting that these two pathways are both involved in intracellular TDP-43 aggregation. Therefore, to determine the mechanisms of intracellular TDP-43 aggregation, we further classified Group 1 genes into microtubule-related proteins (MRPs; PFN1, UBQLN2, TUBA4A, and CHMP2B) and RNA-binding proteins (RBPs; FUS, TAF15, TIA1, and MART3) (Fig. 2B). Notably, the genes encoding RBPs induced TDP-43 co-aggregation only when their mutants were mislocalized into the cytoplasm, suggesting that leakage of RBPs from nuclei is closely associated with the cytoplasmic TDP-43 aggregation.

\section{Liquid-liquid phase separation (LLPS) is involved in RBPs-induced TDP-43 aggregation}

Previous studies reported that FUS and TIA-1, representative RBPs classified into Group 1, form cytoplasmic aggregates through LLPS [23, 24]. Thus, we treated the cells with 1,6-hexanediol (1,6-Hd), an inhibitor of LLPS, to assess the involvement of LLPS in the co-aggregation of RBPs and TDP-43. As shown in Fig. $3 \mathrm{~A}$ and B, 1,6-Hd drastically dissociated the cytoplasmic aggregates of RBPs, mutant FUS and TIA1. Consistent with the decreased number of RBPsinduced aggregates, sequestration of TDP-43 into the aggregates was also significantly prevented (Fig. $3 C$ ). These observations suggest that LLPS drives the cytoplasmic co-aggregation of RBPs and TDP-43. In contrast, 1,6-Hd did not influence either the number of aggregates of MRPs (mutant PFN1 and UBQLN2) or co-aggregates with TDP-43 (Fig. 3A-C), suggesting that a mechanism independent of LLPS drives the co-aggregation of MRPs and TDP-43.

\section{MRPs-induced TDP-43 co-aggregates are sensitive to nocodazole and co-localized with aggresomal markers}

To examine whether the microtubules are essential for MRPs-induced TDP-43 aggregation, we then treated the cells with nocodazole, a microtubule destabilizing reagent. As expected, the nocodazole treatment substantially reduced the amount of co-aggregates of MRPs (PFN1 C71G) and TDP-43 (Fig. 4A, B). Intriguingly, the co-aggregates of MRPs and TDP-43 were co-localized with the aggresomal markers $\gamma$-tubulin (Fig. 4C) and vimentin (Fig. 4D). An aggresome is a peri-nuclear compartment where misfolded proteins accumulate $[25,26]$. Once the cellular protein degradation system is overwhelmed, the misfolded proteins are accumulated into aggresomes through microtubules for a cellular defense. Therefore, our findings suggest that TDP-43 is sequestered into aggregates of MRPs during aggresome formation. Meanwhile, RBPs-induced TDP-43 co-aggregates (FUS ${ }^{\mathrm{P} 525 \mathrm{~L}}$ ) were not affected by nocodazole (Fig. 4A) and did not co-localize with aggresomal markers (Fig. 4C and D), suggesting that MRPs and RBPs sequester TDP-43 into their aggregates through distinct mechanisms.

\section{Histone deacetylase 6 is associated with the co-aggregation of MRPs and TDP-43}

Histone deacetylase 6 (HDAC6) recognizes misfolded proteins and transports them to aggresomes through microtubules [27]. Previous reports showed that aggresomes are involved in the formation of inclusion bodies in the lesions of neurodegenerative diseases such as Huntington's disease [28, 29]. Therefore, we next examined whether the elimination of Hdac6 by siRNA affected TDP-43 co-aggregation with MRPs in N2a cells (Fig. 5A). As shown in Fig. 5B-D, Hdac6 knock-down prevented the aggregation of MRPs as well as TDP-43 co-aggregation with MRPs. Interestingly, relatively small cytoplasmic aggregates of MRPs were still observed in the cells with Hdac6 knock-down (Fig. 5B, asterisks); however, TDP-43 was not co-localized with them. Since HDAC6 is crucial for the transport of misfolded proteins to aggresomes [27, 28], this observation might suggest that TDP-43 is sequestered during compartmentalization. On the other hand, the Hdac6 knock-down did not affect RBPs-induced TDP-43 co-aggregation, indicating that Hdac6 was not involved in the TDP-43 sequestration into the LLPS-induced aggregates.

\section{Different characteristics of TDP-43 inclusions in sporadic ALS suggest multiple pathways form TDP-43 pathology}

The results of TDP-43 co-aggregation obtained by our screening system are based on the overexpression of the inherited ALS/FTLD causative genes. However, it is unclear whether the mechanisms for the formation of TDP-43 aggregation observed in our study are involved in the TDP-43 pathology of sporadic ALS. To examine the involvement of the aggresome pathway in TDP-43 pathology, we examined the co-localization of TDP-43 and HDAC6, an aggresomal marker, in spinal motor neurons of sporadic ALS patients. We found that approximately half of TDP-43 skein-like inclusions were co-localized with HDAC6 (75/160, 46.9\% of total skein-like inclusions) (Fig. 6, Table 2). It should be noted that no co-localization of TDP-43 and HDAC6 was observed in round $(0 / 52)$ or granular (0/33) TDP-43 inclusions. These observations support the hypothesis that TDP-43 skein-like inclusions are, at least partially, formed by a similar mechanism to aggresome formation. Importantly, both the HDAC6-positive and HDAC6-negative inclusions were found in every sporadic ALS patient, suggesting that the mechanisms of TDP-43 aggregation vary in each of the neurons. In conclusion, the different characteristics between skein-like and other types of TDP-43 inclusions suggest that TDP-43 pathology is developed via multiple complexed mechanisms, likely including both aggresome formation and LLPS, in sporadic ALS.

\section{Discussion}

In this study, we demonstrated that: (i) at least two distinct intracellular pathways were involved in TDP-43 cytoplasmic aggregation, including aggresome formation and LLPS; (ii) the two pathways were independent of each other; and (iii) in spinal motor neurons of sporadic ALS, both HDAC6-positive and 
HDAC6-negative aggregates were found in the same ALS patient. Therefore, to the best of our knowledge, we are the first to confirm that HDAC6-dependent aggresome formation is partially involved in skein-like TDP-43 inclusion of sporadic ALS.

Our study revealed that over $70 \%$ of the examined inherited ALS/FTLD causative 19 genes induced TDP-43 co-aggregation in cultured neuronal cells. Our screening system enabled us to assess whether any genes of interest induce cytoplasmic TDP-43 aggregation by a simple transfection-based screening. Moreover, our screening system has been proved useful for investigating the mechanism of TDP-43 aggregation. Indeed, as described above, we identified two distinct mechanisms involved in TDP-43 aggregation by using this system (i.e., aggresome formation and LLPS). Our screening system will also be useful for identifying compounds that prevent cytoplasmic TDP-43 aggregation, which may contribute to a novel therapeutic strategy for ALS/FTLD.

Although our screening system generally well recapitulated cytoplasmic TDP-43 aggregation in ALS/FTLD, our results were partially inconsistent with some previous reports on the pathology of inherited ALS $[17,18]$. Notably, we did not find any evidence of TDP-43 aggregation in the neuronal cells expressing the inherited ALS/FTLD causative genes related to protein degradation (e.g., VCP, UBQLN2, SQSTM1, and TBK1), although the previous studies reported TDP-43 pathology in inherited ALS patients with these mutations [17]. Moreover, the co-localization of TDP-43 and the inherited ALS/FTLD causative gene products are still controversial even though TDP-43 pathology is widely observed in the patients with ALS. For example, some studies have suggested the colocalization of TDP-43 with TIA-1[30] or FUS [31] but other studies do not [32,33]. One possible interpretation of this inconsistency is an experimental limitation of our screening system based on the overexpression of the inherited ALS/FTLD causative genes with a short incubation time before evaluation. Therefore, a careful interpretation is required to compare our results with the inherited ALS/FTLD neuropathology data.

The co-aggregation of TDP-43 and RBPs was sensitive to 1,6-Hd, suggesting that LLPS drives cytoplasmic RBP-induced aggregation. Nucleotides, enriched in nuclei, prevent aggregation driven by LLPS $[34,35]$. Indeed, the previous study demonstrated that an artificially designed RNA, which specifically binds to TDP43, prevents intracellular aggregation of TDP-43 triggered by light-driven forced multimerization of TDP-43 in the cytoplasm [16]. This mechanism could explain our observations that RBPs only aggregated with TDP-43 when they leaked from nuclei into the cytoplasm. On the other hand, the MRPs-induced coaggregation of TDP-43 was dependent on aggresome formation. The role of aggresomes in the accumulation of misfolded proteins has been pointed out in various neurodegenerative diseases [36]. ALS-linked PFN1 mutant proteins that aggregated in the cytoplasm were dependent on microtubules, which was similar to our observations [37]. Intriguingly, these two pathways of TDP-43 aggregation are not complementary and are independent of each other. Inhibiting LLPS prevented RBPs-mediated TDP-43 aggregation, but did not affect MRPs-induced TDP-43 aggregation. Conversely, HDAC6 suppression and microtubular destabilization only affected MRPs-induced TDP-43 aggregation. All these observations indicate that targeting the intracellular mechanism specific to each pathway is key to preventing TDP-43 accumulation. Nonetheless, there is still a possibility that other independent pathways are also involved in cytoplasmic TDP-43 aggregation. Further investigation into the potential mechanisms of TDP-43 accumulation is required.

HDAC6 is a key molecule in aggresome formation. HDAC6 facilitates the transport of misfolded proteins by connecting microtubules and ubiquitinated proteins [27, 36]. The involvement of HDAC6 has been reported in various neurodegenerative diseases such as Huntington's disease [28], Parkinson's disease [27], and Alzheimer's disease [29]. In ALS, it was reported that deletion of Hdac6 ameliorates the disease progression in SOD1-ALS mice [38] and TDP-43 deficiency drastically reduces HDAC6 expression in cultured cells [39]. Moreover, HDAC6 inhibition reversed axonal transports, which are defective in iPSderived motor neurons from ALS patients with FUS mutation [40]. These observations suggest the possibility that HDAC6 is also involved in the ALS pathology similar to other neurodegenerative diseases and that it may be a therapeutic target for ALS through restoring axonal transport. In addition to this, our results strengthen the possibility that inhibition of HDAC6 is a potential therapeutic target for ALS by providing a novel mechanism for preventing microtubule-dependent TDP-43 pathology. HDAC6 inhibition is probably effective for MRPs-linked inherited ALS and partially to sporadic ALS.

Since most of the ALS cases are sporadic, it is important to identify the mechanism responsible for TDP-43 aggregation in the sporadic ALS. Recent reports showed that cytoplasmic TDP-43 was sequestered into aggresome dependent on microtubules in fibroblasts derived from sporadic ALS patients [41]. Consistent with the cited study, we found that TDP-43 was sequestered into aggregates of MRPs in N2a cells and approximately half of TDP-43 skein-like inclusions in sporadic ALS patients were co-localized with HDAC6, an aggresomal marker. Our results suggest that the HDAC6-dependent aggresome pathway may be involved in the formation of skein-like inclusion in ALS/FTLD. However, we also found that both HDAC6-positive and HDAC6-negative skein-like inclusions were observed in the same sporadic ALS patient, as shown in Table 2. In addition, the round and granular inclusions, which are not co-localized with HDAC6, were also found in the same sporadic ALS patient. These observations suggest that the mechanisms responsible for TDP-43 aggregation are different from each other among the remaining motor neurons in the one sporadic ALS patient and that the morphology of TDP-43 inclusions may reflect the corresponding intracellular mechanism. Further studies are needed to determine the mechanism responsible for each type of cytoplasmic TDP-43 aggregation in motor neurons.

\section{Conclusion}

We found that the two distinct pathways, aggresome formation and LLPS, are independently involved in the formation of cytoplasmic TDP-43 inclusions in ALS. Although it is unclear whether each type of cytoplasmic TDP-43 aggregates provokes toxicities to neurons, our findings, together with the other studies, suggest the therapeutic potential of HDAC6 through the several lines of mechanisms in ALS/FTLD.

\section{Abbreviations}

ALS Amyotrophic lateral sclerosis

FTLD Frontotemporal lobar degeneration

HDAC6 histone deacetylase 6 
LLPS liquid-liquid phase separation

MRP microtubule-related protein

RBP RNA binding protein

TDP-43 TAR DNA-binding protein 43

\section{Declarations}

\section{Availability of data and materials}

Data, material, and software information supporting the conclusions of this article are included within the article and its additional files.

\section{Funding}

This work was supported by Grants-in-Aid for Scientific Research 18H02740, 18H04860, 19KK0214 (to KY), and $17 \mathrm{H} 04986$ (to SW) from the Ministry of Education, Culture, Sports, Science and Technology (MEXT), Japan / Japan Society for the Promotion of Science (JSPS), AMED under Grant Number 20ek0109426h0001 (KY), Uehara Memorial Foundation (KY and SW), and the Public Foundation of Chubu Science and Technology Center (SW).

\section{Author's Contribution}

SW and KY designed the study. SW, IH, KO, YM, and SS performed the experiments with support from OK and AS under the supervision of KY. YI and MK organized and provided the ALS/FTLD patient materials. SW and KY interpreted the data and wrote the manuscript. All the authors approved the manuscript.

\section{References}

1. Lee EB, Lee VM, Trojanowski JQ. Gains or losses: molecular mechanisms of TDP43-mediated neurodegeneration. Nat Rev Neurosci. 2011;13:38-50.

2. Ling SC, Polymenidou M, Cleveland DW. Converging mechanisms in ALS and FTD: disrupted RNA and protein homeostasis. Neuron. 2013;79:416-38.

3. Prasad A, Bharathi V, Sivalingam V, Girdhar A, Patel BK. Molecular Mechanisms of TDP-43 Misfolding and Pathology in Amyotrophic Lateral Sclerosis. Front Mol Neurosci. 2019;12:25.

4. Arai T, Hasegawa M, Akiyama H, Ikeda K, Nonaka T, Mori H, Mann D, Tsuchiya K, Yoshida M, Hashizume Y, Oda T. TDP-43 is a component of ubiquitinpositive tau-negative inclusions in frontotemporal lobar degeneration and amyotrophic lateral sclerosis. Biochem Biophys Res Commun. 2006;351:60211.

5. Neumann M, Sampathu DM, Kwong LK, Truax AC, Micsenyi MC, Chou TT, Bruce J, Schuck T, Grossman M, Clark CM, et al. Ubiquitinated TDP-43 in frontotemporal lobar degeneration and amyotrophic lateral sclerosis. Science. 2006;314:130-3.

6. Buratti E. Functional Significance of TDP-43 Mutations in Disease. Adv Genet. 2015;91:1-53.

7. Harrison AF, Shorter J. RNA-binding proteins with prion-like domains in health and disease. Biochem J. 2017;474:1417-38.

8. Courchaine EM, Lu A, Neugebauer KM. Droplet organelles? EMBO J. 2016;35:1603-12.

9. Hyman AA, Weber CA, Julicher F. Liquid-liquid phase separation in biology. Annu Rev Cell Dev Biol. 2014;30:39-58.

10. Luo Y, Na Z, Slavoff SA. P-Bodies: Composition, Properties, and Functions. Biochemistry. 2018;57:2424-31.

11. Protter DSW, Parker R. Principles and Properties of Stress Granules. Trends Cell Biol. 2016;26:668-79.

12. Wolozin B, Ivanov P. Stress granules and neurodegeneration. Nat Rev Neurosci. 2019;20:649-66.

13. Mateju D, Franzmann TM, Patel A, Kopach A, Boczek EE, Maharana S, Lee HO, Carra S, Hyman AA, Alberti S. An aberrant phase transition of stress granules triggered by misfolded protein and prevented by chaperone function. EMBO J 2017.

14. Bolognesi B, Lorenzo Gotor N, Dhar R, Cirillo D, Baldrighi M, Tartaglia GG, Lehner B. A Concentration-Dependent Liquid Phase Separation Can Cause Toxicity upon Increased Protein Expression. Cell Rep. 2016;16:222-31.

15. Asakawa K, Handa H, Kawakami K. Optogenetic modulation of TDP-43 oligomerization accelerates ALS-related pathologies in the spinal motor neurons. Nat Commun. 2020;11:1004.

16. Mann JR, Gleixner AM, Mauna JC, Gomes E, DeChellis-Marks MR, Needham PG, Copley KE, Hurtle B, Portz B, Pyles NJ, et al. RNA Binding Antagonizes Neurotoxic Phase Transitions of TDP-43. Neuron. 2019;102:321-38 e328.

17. Hergesheimer RC, Chami AA, de Assis DR, Vourc'h P, Andres CR, Corcia P, Lanznaster D, Blasco H. The debated toxic role of aggregated TDP-43 in amyotrophic lateral sclerosis: a resolution in sight? Brain 2019, 142:1176-1194.

18. Blokhuis AM, Groen EJ, Koppers M, van den Berg LH, Pasterkamp RJ. Protein aggregation in amyotrophic lateral sclerosis. Acta Neuropathol. 2013;125:777-94.

19. Watanabe S, Hayakawa T, Wakasugi K, Yamanaka K. Cystatin C protects neuronal cells against mutant copper-zinc superoxide dismutase-mediated toxicity. Cell Death Dis. 2014;5:e1497. 
20. Watanabe S, Kaneko K, Yamanaka K. Accelerated disease onset with stabilized familial amyotrophic lateral sclerosis (ALS)-linked mutant TDP-43 proteins. J Biol Chem. 2013;288:3641-54.

21. Motohashi K. A simple and efficient seamless DNA cloning method using SLiCE from Escherichia coli laboratory strains and its application to SLiP sitedirected mutagenesis. BMC Biotechnol. 2015;15:47.

22. Nishino K, Watanabe S, Shijie J, Murata Y, Oiwa K, Komine O, Endo F, Tsuiji H, Abe M, Sakimura K, et al. Mice deficient in the C-terminal domain of TAR DNA-binding protein 43 develop age-dependent motor dysfunction associated with impaired Notch1-Akt signaling pathway. Acta Neuropathol Commun. 2019;7:118.

23. Murray DT, Kato M, Lin Y, Thurber KR, Hung I, McKnight SL, Tycko R. Structure of FUS Protein Fibrils and Its Relevance to Self-Assembly and Phase Separation of Low-Complexity Domains. Cell. 2017;171:615-27 e616.

24. Mackenzie IR, Nicholson AM, Sarkar M, Messing J, Purice MD, Pottier C, Annu K, Baker M, Perkerson RB, Kurti A, et al. TIA1 Mutations in Amyotrophic Lateral Sclerosis and Frontotemporal Dementia Promote Phase Separation and Alter Stress Granule Dynamics. Neuron. 2017;95:808-16 e809.

25. Johnston JA, Ward CL, Kopito RR. Aggresomes: a cellular response to misfolded proteins. J Cell Biol. 1998;143:1883-98.

26. Wang Y, Meriin AB, Zaarur N, Romanova NV, Chernoff YO, Costello CE, Sherman MY. Abnormal proteins can form aggresome in yeast: aggresometargeting signals and components of the machinery. FASEB J. 2009;23:451-63.

27. Kawaguchi Y, Kovacs JJ, McLaurin A, Vance JM, Ito A, Yao TP. The deacetylase HDAC6 regulates aggresome formation and cell viability in response to misfolded protein stress. Cell. 2003;115:727-38.

28. Iwata A, Riley BE, Johnston JA, Kopito RR. HDAC6 and microtubules are required for autophagic degradation of aggregated huntingtin. J Biol Chem. 2005;280:40282-92.

29. Zhang L, Sheng S, Qin C. The role of HDAC6 in Alzheimer's disease. J Alzheimers Dis. 2013;33:283-95.

30. Liu-Yesucevitz L, Bilgutay A, Zhang YJ, Vanderweyde T, Citro A, Mehta T, Zaarur N, McKee A, Bowser R, Sherman M, et al. Tar DNA binding protein-43 (TDP43) associates with stress granules: analysis of cultured cells and pathological brain tissue. PLoS One. 2010;5:e13250.

31. Ikenaka K, Ishigaki S, Iguchi Y, Kawai K, Fujioka Y, Yokoi S, Abdelhamid RF, Nagano S, Mochizuki H, Katsuno M, Sobue G. Characteristic Features of FUS Inclusions in Spinal Motor Neurons of Sporadic Amyotrophic Lateral Sclerosis. J Neuropathol Exp Neurol. 2020;79:370-7.

32. Chen Y, Cohen TJ. Aggregation of the nucleic acid-binding protein TDP-43 occurs via distinct routes that are coordinated with stress granule formation. $J$ Biol Chem. 2019;294:3696-706.

33. Hirsch-Reinshagen V, Pottier C, Nicholson AM, Baker M, Hsiung GR, Krieger C, Sengdy P, Boylan KB, Dickson DW, Mesulam M, et al. Clinical and neuropathological features of ALS/FTD with TIA1 mutations. Acta Neuropathol Commun. 2017;5:96.

34. Maharana S, Wang J, Papadopoulos DK, Richter D, Pozniakovsky A, Poser I, Bickle M, Rizk S, Guillén-Boixet J, Franzmann T, et al: RNA buffers the phase separation behavior of prion-like RNA binding proteins. Science 2018.

35. Kang J, Lim L, Lu Y, Song J. A unified mechanism for LLPS of ALS/FTLD-causing FUS as well as its modulation by ATP and oligonucleic acids. PLoS Biol. 2019;17:e3000327.

36. Richter-Landsberg C, Leyk J. Inclusion body formation, macroautophagy, and the role of HDAC6 in neurodegeneration. Acta Neuropathol. 2013;126:793807.

37. Tanaka Y, Nonaka T, Suzuki G, Kametani F, Hasegawa M. Gain-of-function profilin 1 mutations linked to familial amyotrophic lateral sclerosis cause seeddependent intracellular TDP-43 aggregation. Hum Mol Genet. 2016;25:1420-33.

38. Taes I, Timmers M, Hersmus N, Bento-Abreu A, Van Den Bosch L, Van Damme P, Auwerx J, Robberecht W. Hdac6 deletion delays disease progression in the SOD1G93A mouse model of ALS. Hum Mol Genet. 2013;22:1783-90.

39. Xia Q, Wang H, Zhang Y, Ying Z, Wang G. Loss of TDP-43 Inhibits Amyotrophic Lateral Sclerosis-Linked Mutant SOD1 Aggresome Formation in an HDAC6Dependent Manner. J Alzheimers Dis. 2015;45:373-86.

40. Guo W, Naujock M, Fumagalli L, Vandoorne T, Baatsen P, Boon R, Ordovas L, Patel A, Welters M, Vanwelden T, et al. HDAC6 inhibition reverses axonal transport defects in motor neurons derived from FUS-ALS patients. Nat Commun. 2017;8:861.

41. Lee SM, Asress S, Hales CM, Gearing M, Vizcarra JC, Fournier CN, Gutman DA, Chin LS, Li L, Glass JD. TDP-43 cytoplasmic inclusion formation is disrupted in C9orf72-associated amyotrophic lateral sclerosis/frontotemporal lobar degeneration. Brain Commun. 2019;1:fcz014.

\section{Tables}


Table 1. Results of TDP-43 co-aggregation screening with ALS/FTLD causative genes

\begin{tabular}{|c|c|c|c|c|c|c|c|c|}
\hline Functions & $\begin{array}{l}\text { Gene } \\
\text { symbol }\end{array}$ & Mutations & Localization & Aggregation & $\begin{array}{c}\text { Co- } \\
\text { aggregation } \\
\text { with TDP- } \\
43\end{array}$ & $\begin{array}{l}\text { TDP-43 } \\
\text { inclusions } \\
\text { in } \\
\text { inherited } \\
\text { ALS } \\
\text { cases }\end{array}$ & $\begin{array}{c}\text { Co- } \\
\text { localization } \\
\text { with TDP- } \\
43 \\
\text { inclusions }\end{array}$ & References \\
\hline \multirow[t]{19}{*}{$\begin{array}{l}\text { RNA binding } \\
\text { proteins }\end{array}$} & $T A R D B P$ & wild-type & nuclear & - & - & \multirow{4}{*}{ Yes $^{1-3}$} & \multirow{4}{*}{-} & \multirow{4}{*}{$\begin{array}{l}\text { 1. Pamphlett et al: } \\
\text { Neuropathol Appl } \\
\text { Neurobiol 2009, 35:222. } \\
\text { 2. Van Deerlin et al: LaI } \\
\text { Neurol 2008, 7:409. } \\
\text { 3. Yokoseki et al: Ann } \\
\text { Neurol 2008, 63:538. }\end{array}$} \\
\hline & \multirow[t]{3}{*}{ (ALS10) } & M337V & nuclear & - & - & & & \\
\hline & & G348C & nuclear & - & - & & & \\
\hline & & $\Delta \mathrm{NLS}$ & nuclear & - & - & & & \\
\hline & FUS & wild-type & nuclear & - & - & \multirow{3}{*}{$\mathrm{No}^{1,2}$} & \multirow{3}{*}{$\begin{array}{l}- \\
\text { aYes in } \\
\text { sporadic } \\
\mathrm{ALS}^{3} \text { ) }\end{array}$} & \multirow{3}{*}{$\begin{array}{l}\text { 1. Kwiatkowski et al: } \\
\text { Science 2009, 323:1205. } \\
\text { 2. Vance et } \\
\text { al: Science 2009, 323: } 12 \\
\text { 3. .kenaka et al: J } \\
\text { Neuropathol Exp } \\
\text { Neurol 2020, 79:370. }\end{array}$} \\
\hline & \multirow[t]{2}{*}{ (ALS6) } & R521C & nuclear & - & - & & & \\
\hline & & P525L & cytosol & ++ & ++ & & & \\
\hline & HNRNPA1 & wild-type & nuclear & - & - & \multirow{3}{*}{ Yes } & \multirow{3}{*}{ Yes } & \multirow{3}{*}{$\begin{array}{l}\text { 1. Kim et al: } \\
\text { Nature 2013, 495:467. }\end{array}$} \\
\hline & \multirow[t]{2}{*}{ (ALS20) } & D314V & nuclear & - & - & & & \\
\hline & & N319S & nuclear & - & - & & & \\
\hline & MATR3 & wild-type & nuclear & - & - & \multirow{3}{*}{ Yes $^{1,2}$} & \multirow{3}{*}{ Yes $^{1,2}$} & \multirow{3}{*}{$\begin{array}{l}\text { 1. Johnson et al: Nat } \\
\text { Neurosci 2014, 17:664. } \\
\text { 2. Tada et al: Am J } \\
\text { Pathol 2018, 118: } 507 \text {. }\end{array}$} \\
\hline & \multirow[t]{2}{*}{ (ALS21) } & S85C & nuclear & - & - & & & \\
\hline & & F115C & $\begin{array}{l}\text { nuclear + } \\
\text { cytosol }\end{array}$ & + & + & & & \\
\hline & \multirow[t]{3}{*}{ TAF15 } & wild-type & $\begin{array}{l}\text { nuclear + } \\
\text { cytosol }\end{array}$ & + & ++ & \multirow{3}{*}{$\begin{array}{l}\text { Not } \\
\text { reported }\end{array}$} & \multirow{3}{*}{-} & \\
\hline & & G391E & $\underset{\text { cytosol }}{\text { nuclear }}+$ & + & ++ & & & \\
\hline & & R408C & $\begin{array}{c}\text { nuclear }+ \\
\text { cytosol }\end{array}$ & + & + & & & \\
\hline & \multirow[t]{3}{*}{ TIA1 } & wild-type & $\begin{array}{c}\text { nuclear + } \\
\text { cytosol }\end{array}$ & + & ++ & & & \multirow{3}{*}{$\begin{array}{l}\text { 1. Hirsch-Reinshagen et } \\
\text { Acta Neuropatho } \\
\text { Commun 2017, 5:96 } \\
\text { 2. Mackenzie et } \\
\text { al: Neuron 2017, 16:95. }\end{array}$} \\
\hline & & P362L & $\begin{array}{l}\text { nuclear }+ \\
\text { cytosol }\end{array}$ & + & + & Yes $^{1,2}$ & $\mathrm{Yes}^{1} / \mathrm{No}^{2}$ & \\
\hline & & A381T & $\begin{array}{c}\text { nuclear + } \\
\text { cytosol }\end{array}$ & + & + & & & \\
\hline \multirow[t]{9}{*}{$\begin{array}{l}\text { Intracellular } \\
\text { trafficking }\end{array}$} & FIG4 & wild-type & cytosol & + & + & \multirow{3}{*}{$\begin{array}{l}\text { Not } \\
\text { reported }\end{array}$} & \multirow{3}{*}{-} & \\
\hline & \multirow[t]{2}{*}{ (ALS11) } & Q403X & cytosol & + & + & & & \\
\hline & & V654A & cytosol & + & + & & & \\
\hline & $C H M P 2 B$ & wild-type & cytosol & + & + & \multirow{3}{*}{ No } & \multirow{3}{*}{-} & \\
\hline & (ALS17) & $\mathrm{I} 29 \mathrm{~V}$ & cytosol & + & + & & & $\begin{array}{l}\text { 1. Cairns et al: } A m J \\
\text { Pathol 2007, 171: } 227 \text {. }\end{array}$ \\
\hline & & Q206H & $\begin{array}{c}\text { nuclear + } \\
\text { cytosol }\end{array}$ & + & + & & & \\
\hline & $T F G$ & wild-type & cytosol & +++ & ++ & & & \\
\hline & & G269V & cytosol & +++ & ++ & $\begin{array}{l}\text { Not } \\
\text { reported }\end{array}$ & - & \\
\hline & & P285L & cytosol & +++ & ++ & & & \\
\hline $\begin{array}{l}\text { Protein } \\
\text { degradation }\end{array}$ & $V C P$ & wild-type & cytosol & - & - & & $\begin{array}{l}\text { Not } \\
\text { reported }\end{array}$ & $\begin{array}{l}\text { 1. Cairns et al: } A m J \\
\text { Pathol 2007, 171: } 227 \text {. } \\
\text { 2. Neumann et al: } J\end{array}$ \\
\hline & (ALS14) & R155H & cytosol & - & - & Yes $^{1-3}$ & $\begin{array}{l}\text { (Yes in } \\
\text { cultured }\end{array}$ & $\begin{array}{l}\text { Neuropahol Exp } \\
\text { Neurol 2007, 66:152. }\end{array}$ \\
\hline & & A232E & cytosol & - & - & & cells $^{3}$ ) & $\begin{array}{l}\text { 3. Gitcho et al: J10I } \\
\text { Chem 2009, 284:12384. }\end{array}$ \\
\hline & $\begin{array}{l}U B Q L N 2 \\
\text { (ALS15) }\end{array}$ & $\begin{array}{c}\text { wild-type } \\
\text { P497H }\end{array}$ & $\begin{array}{l}\text { cytosol } \\
\text { cytosol }\end{array}$ & $\begin{array}{l}+++ \\
+++\end{array}$ & $\begin{array}{l}++ \\
++\end{array}$ & Yes $^{1-3}$ & $\begin{array}{c}\text { Yes }^{3} \\
\text { पYes in }\end{array}$ & $\begin{array}{l}\text { 1. Deng et al: } \\
\text { Nature 2011, 477: } 211 \text {. } \\
\text { 2. Williams et al: Neuro. } \\
\text { Aging 2012, 33:2527. }\end{array}$ \\
\hline
\end{tabular}




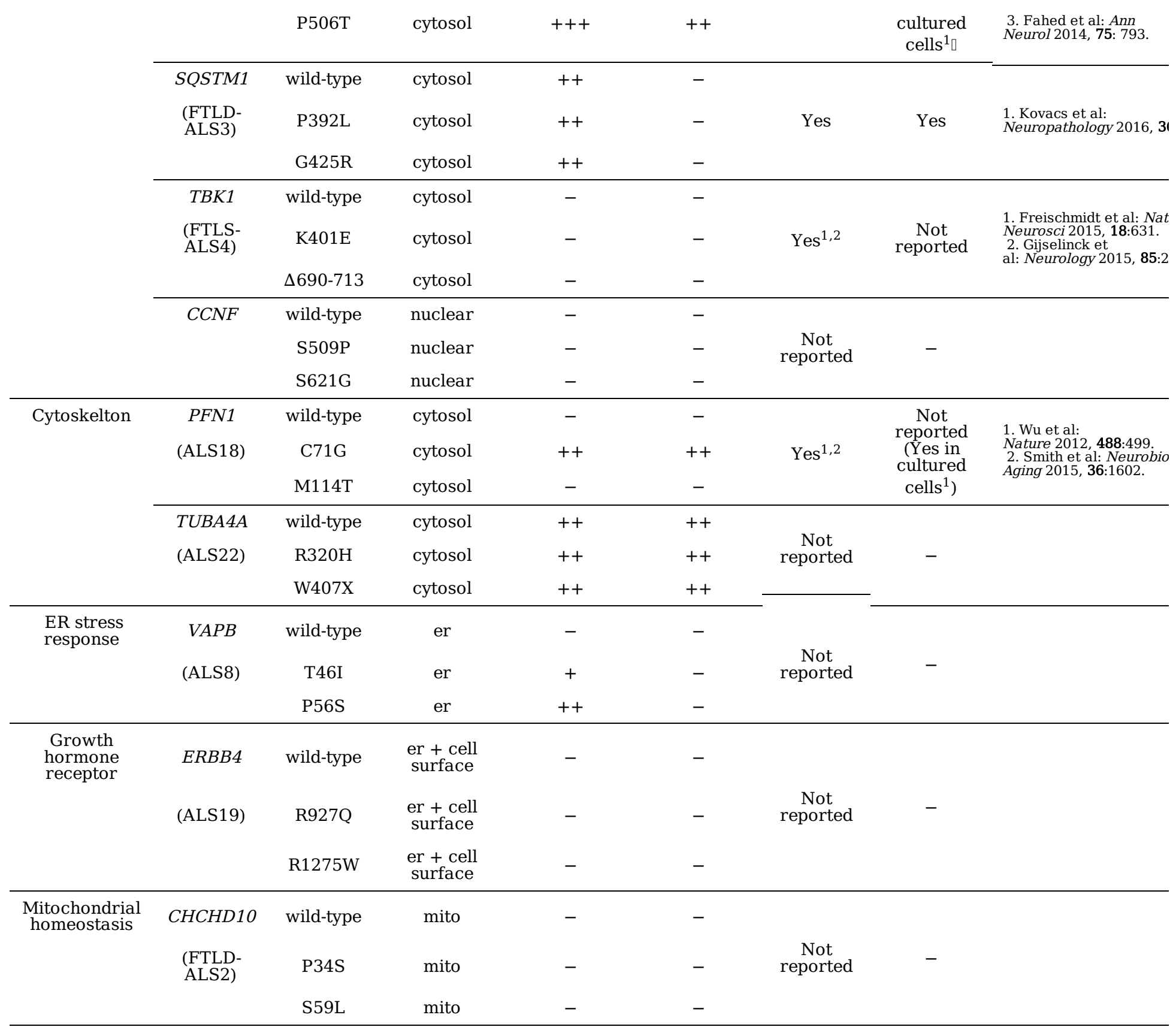

"Aggregation" was evaluated by ratio with intracellar aggregates of each gene product. +: mild (< $30 \%)$, ++: medium (30-70\%), +++: severe (> $70 \%)$

"Co-aggregation with TDP-43" was evaluated co-localization of TDP-43 aggregates and aggregates of each gene product. +: partially, ++: completely.

More than 50 cells were counted in each trial $(\mathrm{n}=3$ each).

er: endoplasmic reticullum, mito: mitochondria 
Table 2 Co-localization of TDP-43 and HDAC6 in sporadic ALS motor neurons

\begin{tabular}{ccccr}
\hline \multirow{2}{*}{ Patient } & \multirow{2}{*}{ Tissue } & Skein-like & Round & Granular \\
\cline { 3 - 5 } & & \multicolumn{2}{c}{ HDAC6(+)/Total (percentage) } \\
\hline sALS \#1 & CSC & $21 / 32(65.6)$ & $0 / 4(0)$ & $0 / 4(0)$ \\
sALS \#2 & CSC & $29 / 38(76.3)$ & $0 / 5(0)$ & $0 / 7(0)$ \\
sALS \#3 & LSC & $6 / 27(22.2)$ & $0 / 34(0)$ & $0 / 9(0)$ \\
SALS \#4 & LSC & $3 / 34(8.8)$ & $0 / 2(0)$ & $0 / 13(0)$ \\
sALS \#5 & LSC & $13 / 16(81.3)$ & $0 / 5(0)$ & $0 / 0(0)$ \\
sALS \#6 & LSC & $3 / 13(23.1)$ & $0 / 2(0)$ & $0 / 0(0)$ \\
\hline Total & & $75 / 160(46.9)$ & $0 / 52(0)$ & $0 / 33(0)$ \\
\hline
\end{tabular}

CSC: cervical spinal cord, LSC: lumber spinal cord

\section{Figures}

A

mCherry fused ALS/FTLD-causative gene

expression plasmids

Transfection

2 Differentiation
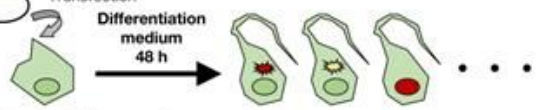

Neuro2a cells stably expressing

aggregation/co-aggregation of EGFP/mCherry

Control

\section{B}

mCherry $\underset{- \text { TDPFP }}{\text { MANLS }}$ Merged
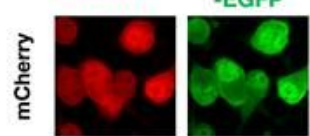

C Group 1: Co-aggregate with TDP-43

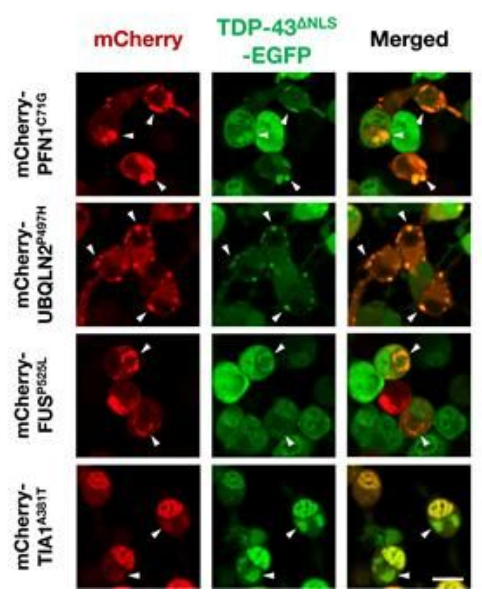

D Group 2: Aggregate without TDP-43

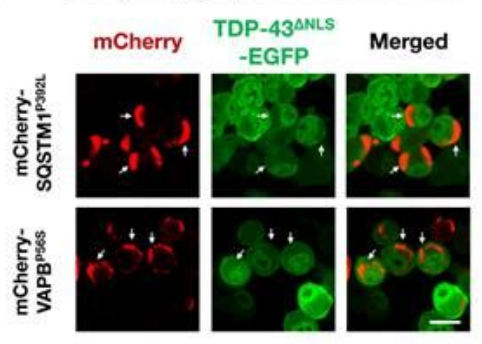

E Group 3: Do not aggregate

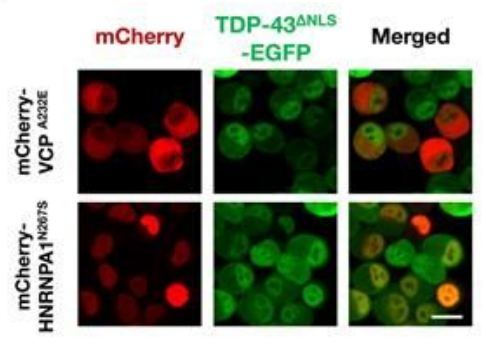

Figure 1

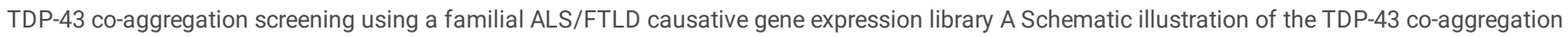

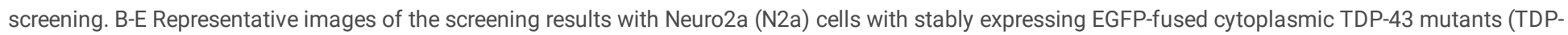

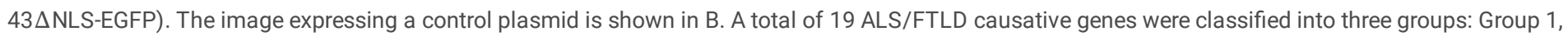

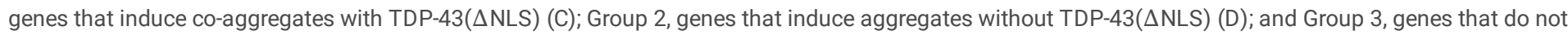

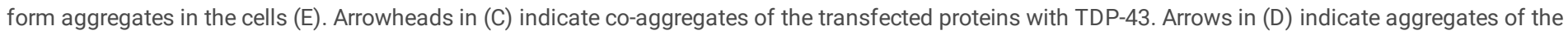
transfected proteins excluding TDP-43. Scale bars $=20 \mu \mathrm{m}$. 


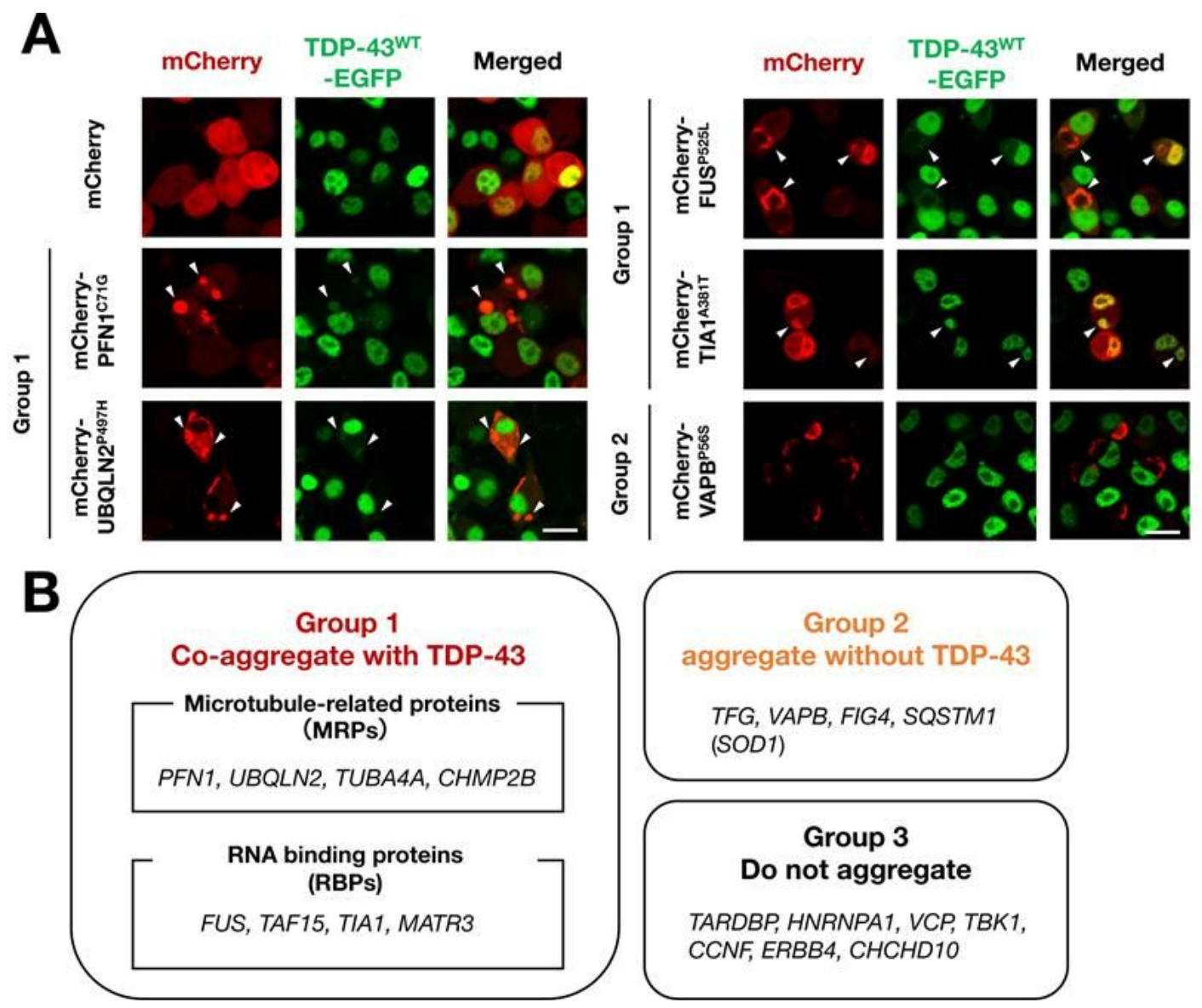

Figure 2

The ALS/FTLD causative genes co-aggregated with TDP-43 induces cytoplasmic translocation of wild-type TDP-43 A Representative images of the screening results with N2a cells stably expressing EGFP-fused wild-type TDP-43 (TDP-43WT). Arrowheads indicate cytoplasmic co-aggregates of the Group 1 gene products and TDP-43WT. Scale bars $=20 \mu \mathrm{m}$. B The summary of the TDP-43 co-aggregation screening. The genes in Group 1 were further classified into two subgroups based on their physiological functions: microtubule-related proteins (MRPs) and RNA-binding proteins (RBPs). 
A

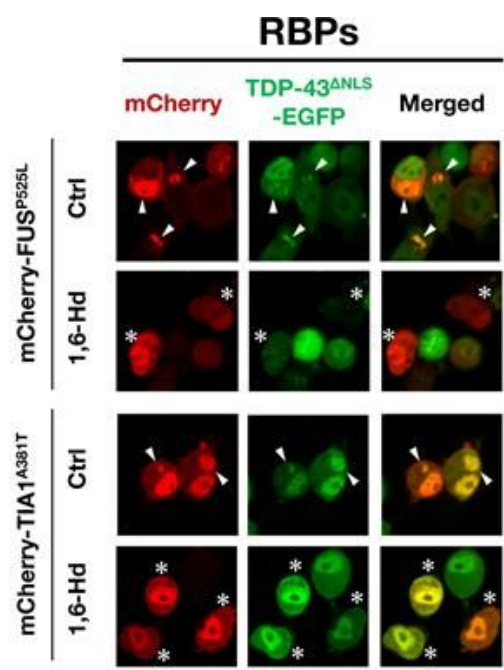

B
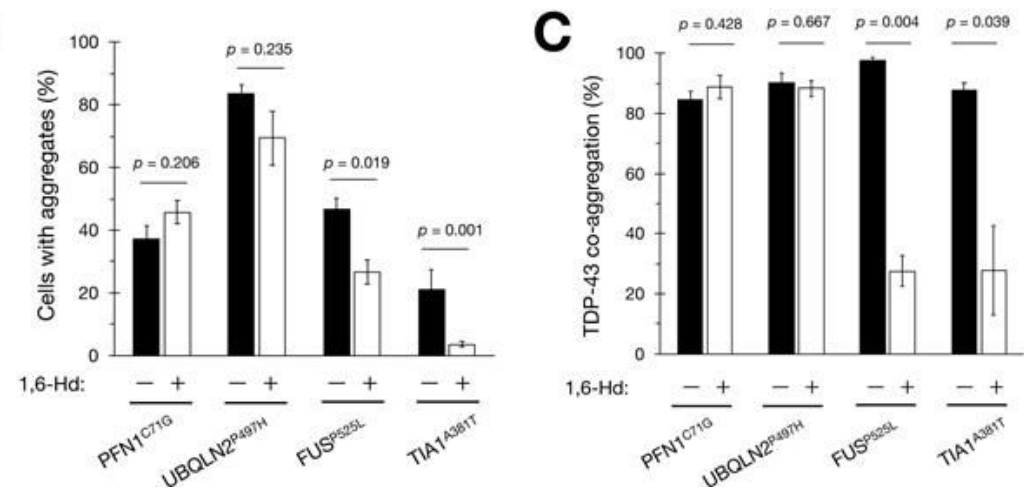

Figure 3

Liquid-liquid phase separation (LLPS) is involved in RBPs-induced TDP-43 co-aggregation Administration of 1,6-hexanediol (1,6-Hd), an inhibitor of LLPS, dissociated TDP-43 co-aggregates with RBPs (FUS and TIA1) but not with MRPs (PFN1 and UBQLN2) in N2a-TDP-43 $\Delta$ NLS cells. Representative images are shown in A. Both the ratio of the cells with TDP-43 aggregates (B) and TDP-43 co-aggregation (C) were reduced, specifically in the cells expressing RBPs with 1,6-Hd. Arrowheads indicate co-aggregates of the RBPs and MAPs with TDP-43 $\Delta$ NLS. Whereas, asterisks indicate TDP-43 $\Delta$ NLS cells without co-aggregates of the RBPs by the 1,6-Hd treatment. More than 50 cells in each condition were analyzed for the quantification. Data are expressed as mean \pm SEM $(n=3)$. 


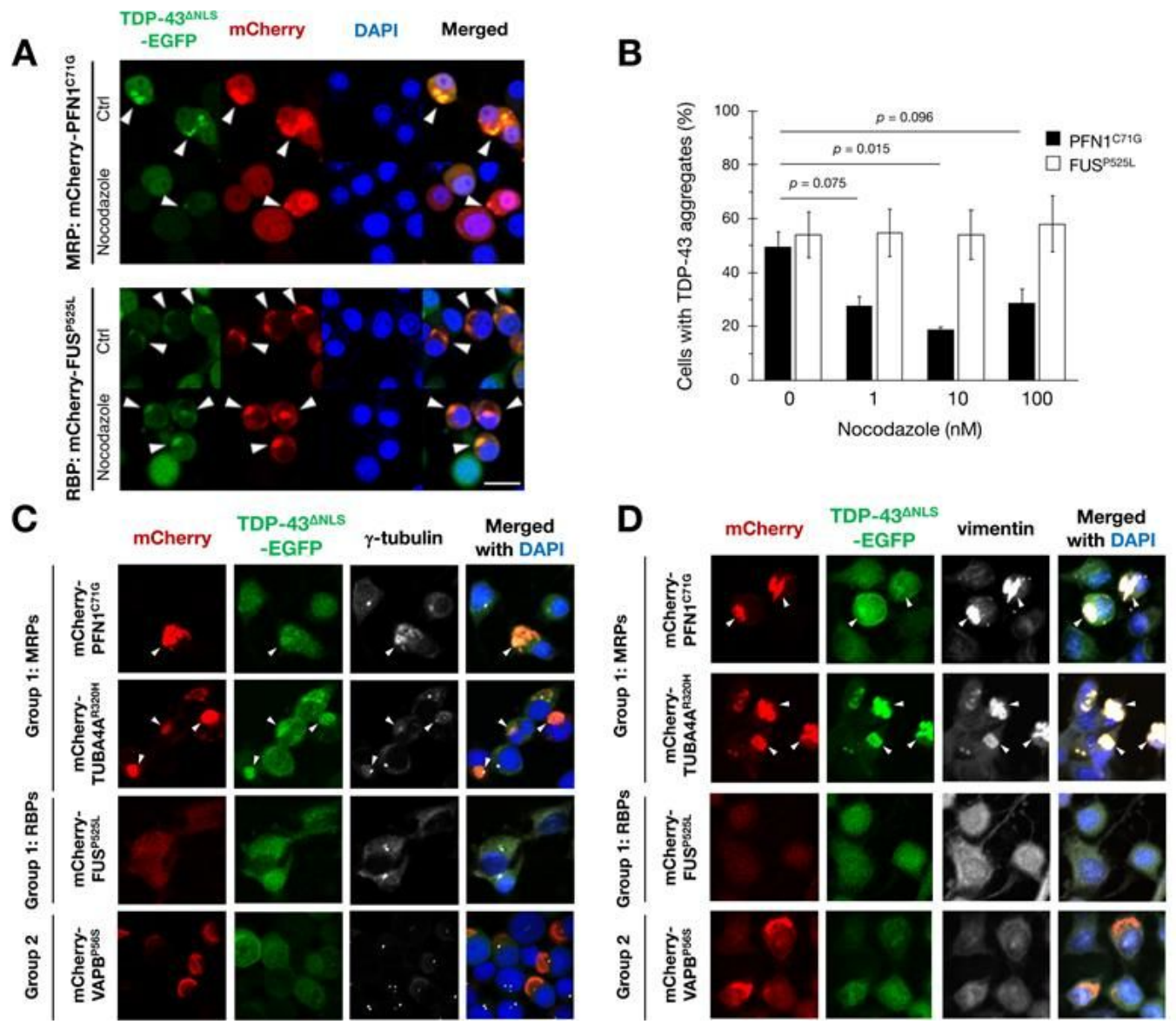

Figure 4

MRPs-induced TDP-43 aggregates are dependent on microtubules and co-localized with aggresome markers A and B Nocodazole, a microtubule destabilizing reagent, dissociated TDP-43 co-aggregates with the MRPs (PFN1) but not with the RBPs (FUS). Arrowheads indicate co-aggregates of the MRPs/RBPs and TDP-43 $\Delta$ NLS (A). The ratio of the cells with TDP-43 aggregates was quantified by counting more than 100 cells in each trial (B). Data are expressed as mean \pm SEM $(n=3)$. C and D Representative immunofluorescence images showed that the MRPs (PFN1 and TUBA4A) induced TDP-43 co-aggregates were colocalized with the aggresomal markers $y$-tubulin (C) and vimentin (D). Arrowheads indicate the co-localization of MRPs, TDP-43, and $y$-tubulin in $C$ or vimentin in D. Note that there were no aggregates in RBPs, represented by FUS, because the aggregates of RBPs were not observed in this immunocytochemical condition. Scale bars $=20 \mu \mathrm{m}$. 
A

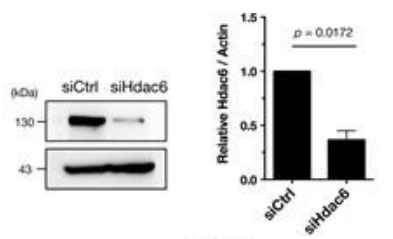

B
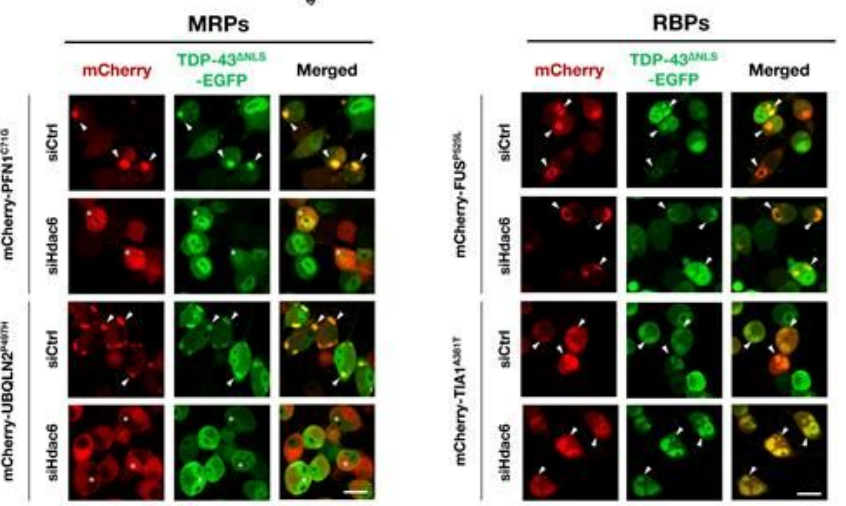

C
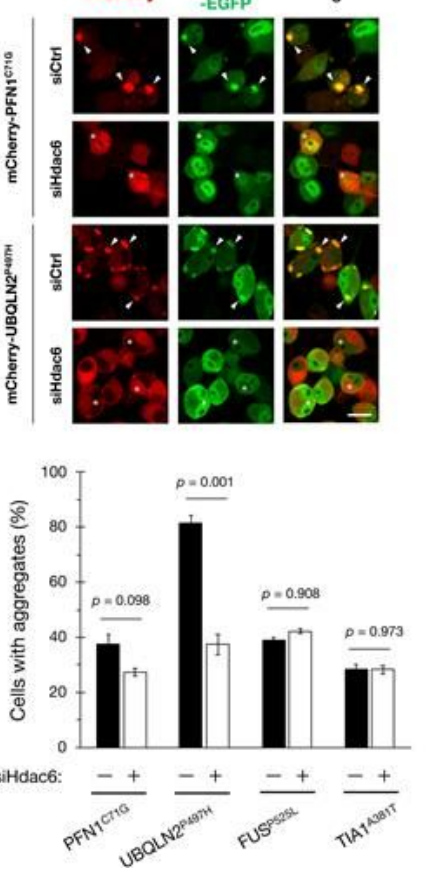

D

$\rho=0.002 \quad \rho=0.005 \quad \rho=0.317 \quad \rho=0.490$

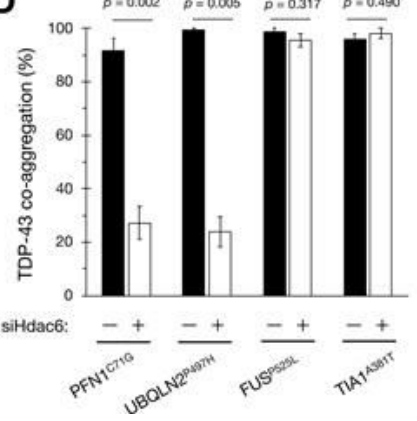

Figure 5

Histone deacetylase 6 (Hdac6) is involved in MRPs-induced TDP-43 co-aggregation A Confirmation of Hdac6 knock-down in N2a cells by the treatment with siRNA (siHdac6). The expression levels relative to the control siRNA treated samples (siCtrl) were shown. Data are expressed as mean \pm SEM $(n=3)$. B-D Hdac6 suppression specifically prevented MRPs (PFN1 or UBQLN2)-induced TDP-43 co-aggregation but not RBPs (FUS or TIA1). Representative images are shown in B. Arrowheads indicate co-aggregates of the MRPs and RBPs with TDP-43 NLS. Whereas, asterisks indicate TDP-43 $\Delta$ NLS cells without coaggregates of the MRPs by Hdac6 suppression. The ratio of the cells with MRPs aggregates was reduced when the cells were treated with siHdac6 (C). Similarly, the ratio of MRPs and TDP-43 co-aggregation was also substantially reduced, specifically in the cells treated with siHdac6 (D). More than 50 cells in each condition were analyzed for the quantification. Data are expressed as mean \pm SEM $(n=3)$. 


\section{TDP-43 HDAC6 DAPI Merged}

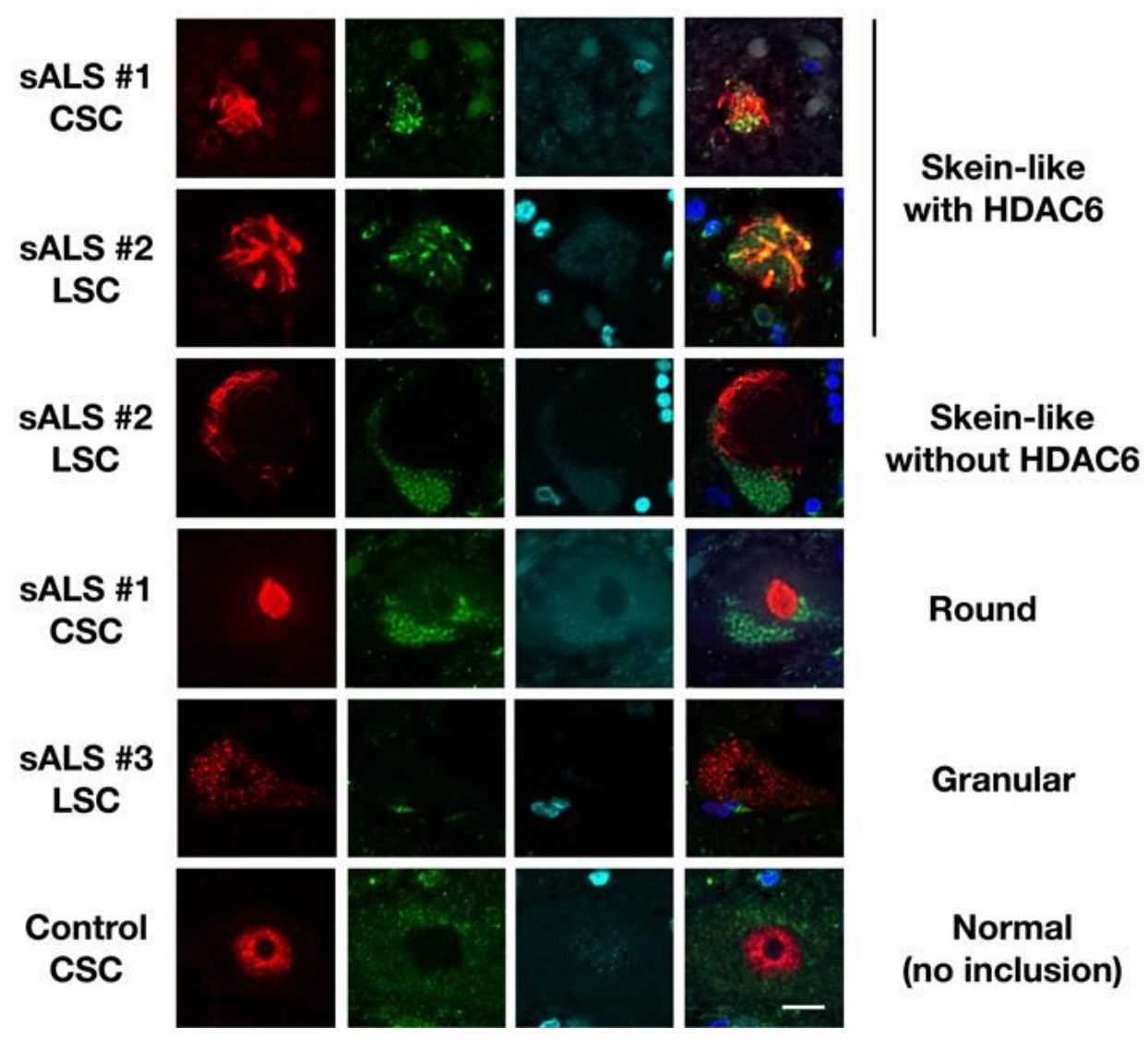

Figure 6

A part of skein-like cytoplasmic inclusion of TDP-43 co-localized with HDAC6 in the spinal motor neurons of sporadic ALS patients. Representative immunofluorescent images of the spinal cord sections of sporadic ALS patients stained with TDP-43, HDAC6, and DAPI demonstrate various TDP-43immunopositive inclusions. The summary of the quantification is shown in Table 2. Note that only the skein-like inclusions of TDP-43 co-localized with HDAC6. Scale bar $=20 \mu \mathrm{m}$.

\section{Supplementary Files}

This is a list of supplementary files associated with this preprint. Click to download.

- AdditionalFile120200508swClear.docx

- AdditionalFile120200508swClear.docx 